01;03

\title{
Математическое моделирование возникновения и развития кавитации в турбулентном потоке жидкости в симметричном канале
}

\author{
() У. Ибен ${ }^{1}$, А.В. Махнов ${ }^{2, \uparrow, ~ А . A . ~ Ш м и д т ~}{ }^{2,3}$ \\ ${ }^{1}$ Robert Bosch GmbH, Corporate Research, St. Petersburg, Russia \\ ${ }^{2}$ Санкт-Петербургский политехнический университет Петра Великого, Санкт-Петербург, Россия \\ ${ }^{3}$ Физико-технический институт им. А.Ф. Иофрфе РАН, Санкт-Петербург, Россия \\ ๑E-mail: a_makhnov@mail.ru
}

Поступило в Редакцию 9 февраля 2018 г.

Исследованы процессы возникновения и развития кавитации при течении углеводородного топлива в микроканале квадратного сечения под действием постоянного большого перепада давления. Показано, что течение является существенно нестационарным и, несмотря на то что канал имеет вертикальную и горизонтальную плоскости симметрии, структура кавитирующего течения является существенно несимметричной. Это обстоятельство играет важную роль при анализе фундаментальной проблемы возникновения кавитации и решении широкого круга прикладных задач. Численное моделирование турбулентных кавитационных течений основано на уравнениях Навье-Стокса, дополненных уравнением состояния баротропной среды. При проведении расчетов использован модифицированный алгоритм открытой вычислительной среды OpenFOAM. Сопоставление результатов тестовых расчетов с экспериментальными данными продемонстрировало адекватность и эффективность разработанного алгоритма.

DOI: 10.21883/PJTF.2019.02.47223.17249

Исследования кавитации играют важную роль при решении широкого круга прикладных проблем: от задач высокоскоростного движения тел в жидкости [1] до проблем технологии обработки материалов [2]. Исследования кавитации важны также с точки зрения развития фундаментальных представлений механики гетерогенных сред (см., например, [3-5]).

В настоящей работе рассматривается гидродинамическая кавитация. Это означает, что фазовый переход жидкость-пар происходит в высокоскоростном потоке жидкости в локальных областях, в которых давление падает ниже давления насыщенных паров. В таких потоках значения числа Рейнольдса обычно соответствуют турбулентному режиму, что необходимо учитывать при моделировании. Турбулентность является одним из основных факторов, влияющих на развитие течения и как следствие на возникновение и развитие кавитации, поскольку локальные области низкого давления могут возникать и в турбулентных вихрях.

Ключевым вопросом исследования течений кавитирующих жидкостей является выбор модели возникновения и развития кавитации. Существующие модели гомогенной и гетерогенной нуклеации паровых пузырей (см., например, [6]) требуют больших вычислительных ресурсов. Одним из альтернативных подходов к описанию состояния кавитирующей жидкости является использование моделей равновесной квазигомогенной баротропной смеси (см., например, [7]).

Математическая модель, используемая в рамках данного исследования, включает в себя уравнения Навье-Стокса (уравнения сохранения массы и импульca, см. далее) в комбинации с уравнениями модели турбулентности и модели равновесной квазигомогенной баротропной смеси жидкости и пара. Для описания турбулентности используется перспективный вихреразрешающий подход IDDES (improved delayed detached eddy simulation) [8], который является комбинацией подхода, основанного на осредненных уравнениях Навье-Стокса (Reynolds averaged Navier-Stokes, RANS), и метода крупных вихрей (large eddy simulation, LES). При этом линейный масштаб турбулентности $l_{\text {IDDES }}$ определяется как

$$
l_{\mathrm{IDDES}}=\tilde{f}_{d}\left(1+f_{e}\right) l_{\mathrm{RANS}}+\left(1-\tilde{f}_{d}\right) l_{\mathrm{LES}},
$$

где $\tilde{f}_{d}$ и $f_{e}-$ эмпирические функции, предназначенные для координации работы RANS- и LES-ветвей метода IDDES; $l_{\text {RANS }}$ и $l_{\text {LES }}$ - линейные масштабы турбулентности RANS- и LES-моделей соответственно.

Для описания фазового перехода в процессе кавитации используется уравнение состояния равновесной квазигомогенной баротропной смеси жидкости и паpa [7], которое комбинирует модель „слабо сжимаемой жидкости“ и модель совершенного пара.

Предполагая адиабатичность системы и пренебрегая изменением энтропии из-за диссипации, массовую долю пара, образующегося при падении давления жидкости ниже давления насыщенных паров, можно записать как

$$
m(p) \approx-\frac{1}{r_{\text {evap }}}\left(\frac{d h^{\prime}}{d p}-\frac{1}{\rho_{0}}\right)\left(p-p_{v}\right) \approx-K\left(p-p_{v}\right) .
$$

Здесь $m$ - массовая доля пара, $p_{v}$ - давление насыщенных паров, $p$ - локальное значение давления, $\rho_{0}-$ плотность жидкости в условиях насыщения, $r_{\text {evap }}-$ удельная энтальпия парообразования, $h^{\prime}-$ удельная энтальпия жидкой фазы. Коэффициент $K$ считается постоянным. 


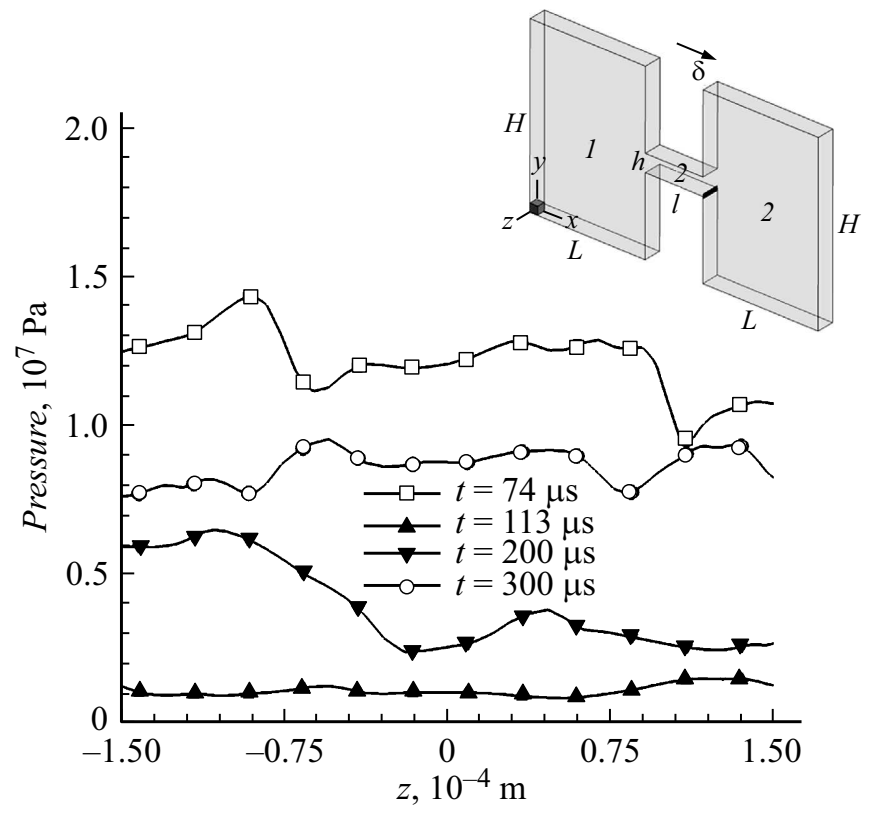

Рис. 1. Профили давления поперек канала при $x=3 \mathrm{~mm}$, $y=1.35 \mathrm{~mm}$ для различных моментов времени. В правом верхнем углу показана схема канала: $L=2 \mathrm{~mm}$, $l=1 \mathrm{~mm}, \quad H=3 \mathrm{~mm}, \quad h=0.3 \mathrm{~mm}, \quad \delta=0.3 \mathrm{~mm}$. Область $1-p=3 \cdot 10^{7} \mathrm{~Pa}, T=353 \mathrm{~K}, \rho=800.32 \mathrm{~kg} / \mathrm{m}^{3}$; область $2-$ $p=1.14 \cdot 10^{7} \mathrm{~Pa}, T=353 \mathrm{~K}, \rho=786.2 \mathrm{~kg} / \mathrm{m}^{3}$.

Таким образом, полная система уравнений может быть записана в виде

$$
\begin{gathered}
\frac{\partial \rho}{\partial t}+\nabla \cdot(\rho \mathbf{u})=0, \\
\frac{\partial}{\partial t}(\rho \mathbf{u})+\nabla \cdot(\rho \mathbf{u u})-\nabla \cdot(\mu \nabla \mathbf{u})=-\nabla p, \\
\frac{\partial(\rho \tilde{v})}{\partial t}+\frac{\partial\left(\rho u_{k} \tilde{v}\right)}{\partial x_{k}}=\rho P^{v}-\rho D^{v} \\
+\frac{\rho}{\sigma}\left\{\frac{\partial}{\partial x_{k}}\left[(v+\tilde{v}) \frac{\partial \tilde{v}}{\partial x_{k}}\right]\right. \\
\left.+c_{b 2}\left[\left(\frac{\partial \tilde{v}}{\partial x_{k}}\right)\left(\frac{\partial \tilde{v}}{\partial x_{k}}\right)\right]\right\}+\rho f_{t 1} \Delta U, \\
\rho(p)= \begin{cases}\rho_{0}+\frac{1}{c_{L}^{2}}\left(p-p_{v}\right), & p \geqslant p_{v}, \\
\frac{m(p)}{p /\left(R_{v} T_{0}\right)}+\frac{1-m(p)}{\rho_{0}}, & 0<p<p_{v} .\end{cases}
\end{gathered}
$$

Первые два члена в правой части уравнения (5) отвечают за генерацию и диссипацию турбулентности соответственно.

Температура смеси $T_{0}$ и скорость звука в жидкой фазе $c_{L}$ считаются постоянными, $R_{v}-$ газовая постоянная для пара.

Через $\rho, \mathbf{u}$ и $p$ обозначены поля плотности, скорости и давления смеси соответственно. Динамический коэффициент вязкости смеси определяется как $\mu=\rho(v+\tilde{v})$, где $v$ и $\tilde{v}-$ кинематические коэффициенты молекулярной и турбулентной вязкости соответственно.

Исследовалось течение жидкости, моделирующей углеводородное топливо, под действием большого перепада давления в канале квадратного сечения. При рассматриваемых условиях давление насыщенных паров модельной жидкости равно $9745.4 \mathrm{~Pa}$, плотность жидкости в условиях насыщения $777.56 \mathrm{~kg} / \mathrm{m}^{3}$, кинематический коэффициент молекулярной вязкости $1.8465 \cdot 10^{-6} \mathrm{~m}^{2} / \mathrm{s}$. Схема и размеры канала представлены на рис. 1. На входе и выходе из канала давление считается постоянным и равным $3 \cdot 10^{7}$ и $1.14 \cdot 10^{7} \mathrm{~Pa}$ соответственно.

Расчеты показали, что кавитирующее течение в канале имеет сложную пространственную нестационарную структуру, что видно, в частности, из рис. 1, на ко-

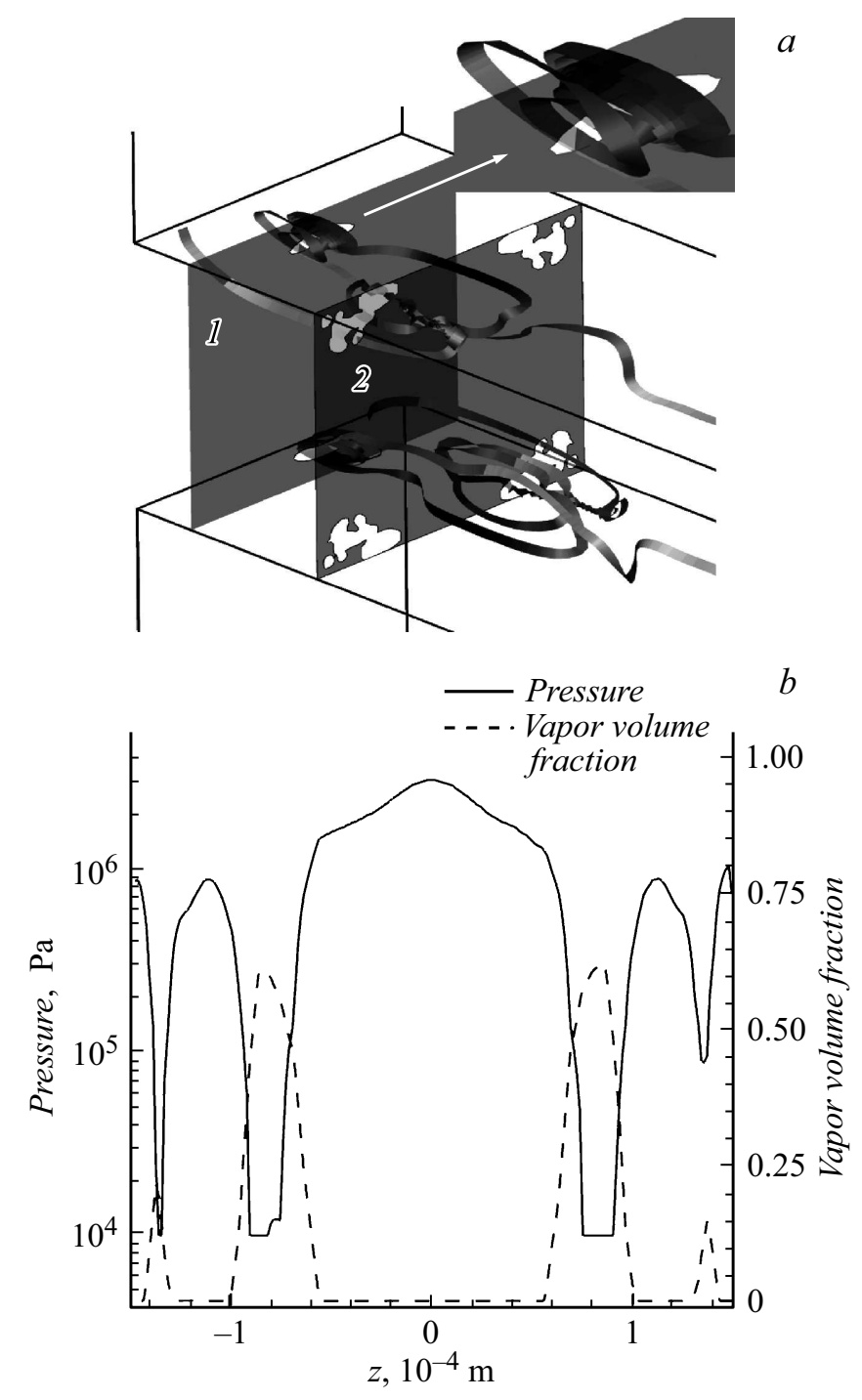

Рис. 2. $a-$ возникновение и развитие кавитации в вихревых зонах, образующихся при отрыве потока от верхней и нижней входных кромок канала (поперечные сечения канала: 1 $x=2.07 \mathrm{~mm}, 2-x=2.19 \mathrm{~mm}) ; b-$ профили давления и объемной доли пара поперек канала при $x=2.19 \mathrm{~mm}$, $y=1.64 \mathrm{~mm}$ в момент возникновения кавитации $(t=31 \mu \mathrm{s})$. 

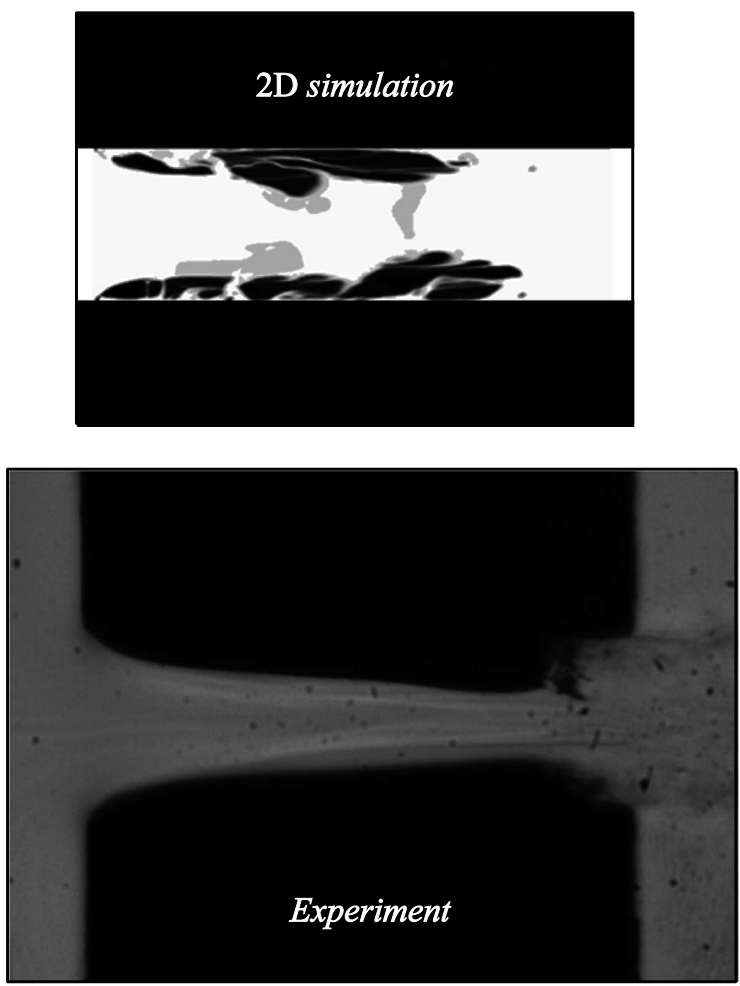
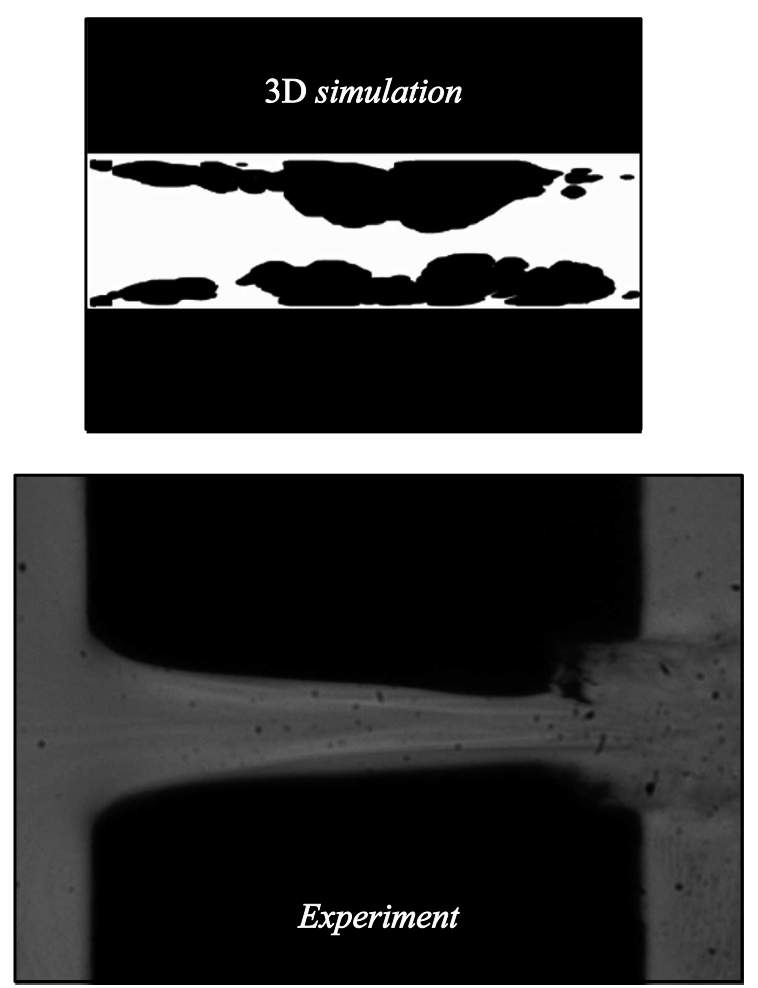

Рис. 3. Сравнение распределений объемной доли паровой фазы, полученных при моделировании и в экспериментах.

тором представлены профили давления вдоль нижнего ребра выходного сечения канала в условиях развитой кавитации (ребро показано темной линией на схеме). Отсутствие симметрии профилей давления и их сильная зависимость от времени связаны со сложной неоднородной структурой отрывного потока, в котором содержатся макроскопические паровые полости. В зависимости от параметров течения они могут расти или коллапсировать, что и вызывает резкие изменения давления, которые в свою очередь приводят к эрозионному разрушению.

Для понимания механизмов развития кавитации в рассматриваемом потоке на рис. 2, $a$ приведены две характерные линии тока отрывного течения. Видно, что возникновение кавитации (светлые области в сечении 1) соответствует областям наибольшей завихренности потока (см. увеличенный фрагмент рис. 2,a). Эти соображения подтверждаются также сравнением профилей давления и объемной доли паровой фазы вдоль линии, проходящей через кавитационные каверны в сечении 2, которые приведены на рис. $2, b$. Как и следует из принятой модели, возникновение кавитации соответствует падению давления жидкости ниже давления насыщенных паров. Падение давления связано с отрывом потока от верхней и нижней входных кромок канала и возникновением вихревых структур в отрывных зонах. Дальнейшее развитие этих структур и кавитационных зон приводит к нарушению симметрии потока. Это становится особенно заметным при сравнении результатов расчетов с экспериментальными данными.

Такое сравнение представлено на рис. 3, где приведены экспериментальные данные, полученные на установке, описанной в [7] (шлирен-фотографии распределения объемной доли паровой фазы в канале), и результаты двумерного (в пренебрежении эффектами боковых стенок и несимметричности течения относительно вертикальной осевой плоскости) и трехмерного численного моделирования. В последнем случае приведен результат осреднения пространственного распределения паровой фазы по координате $z$. Видно, что в рассмотренном случае двумерные расчеты не позволяют получить удовлетворительное совпадение с экспериментальными данными по форме, размеру и расположению кавитационных зон. Результаты трехмерных расчетов существенно ближе к экспериментальным данным. Этот результат важен для анализа возникновения кавитации и оценки возможности развития кавитационной эрозии.

Таким образом, проведенные исследования продемонстрировали работоспособность сформулированной модели и показали, что течение кавитирующей жидкости в каналах, обладающих симметрией, имеет сложный пространственный и нестационарный характер. Это необходимо учитывать при определении силовых нагрузок на элементы конструкций и эрозионной стойкости таких каналов, что имеет важное прикладное значение.

A.В. Махнов благодарит Научный центр G-RISC и германскую службу академических обменов за поддержку. 


\section{Список литературы}

[1] Федоров С.В., Велданов В.А. // ЖТФ. 2013. Т. 83. В. 2. С. 15 20.

[2] Столяр С.В., Баюков О.А., Исхаков Р.С., Ярославцев Р.Н., Ладыгина В.П. // Письма в ЖТФ. 2017. Т. 43. В. 24. С. 3-8.

[3] Franc J.-P., Michel J.-M. Fundamentals of cavitation // Series on fluid mechanics and its applications. V. 76 / Ed. R. Moreau. Kluwer Academic Publ., 2004. 321 p.

[4] Волков Г.А., Петров Ю.В., Груздков А.А. // ЖТФ. 2015. T. 85. B. 5. C. $123-126$

[5] Тесленко В.С., Дрожжжин А.П., Медведев Р.Н. // Письма в ЖТФ. 2014. Т. 40. В. 22. С. 76-82.

[6] Chernyshev A., Kitanin E., Kumzerova E., Schmidt A. Numerical simulation of degassing liquid flow in tube // Proc. of the 6th Int. Conf. on multiphase flow (ICMF-2007). Leipzig, Germany, 2007. Paper N PS6-39.

[7] Skoda R., Iben U., Morozov A., Mihatsch M., Schmidt S.J., Adams N.A. Numerical simulation of collapse induced shock dynamics for the prediction of the geometry, pressure and temperature impact on the cavitation erosion in micro channels // WIMRC 3rd Int. Cavitation Forum. University of Warwick, UK, 2011. DOI: 10.13140/2.1.2676.9287

[8] Shur M.L., Spalart P.R., Strelets M.K., Travin A.K. // Int. J. Heat Fluid Flow. 2008. V. 29. P. 1638-1649. 